\title{
High microphone signal-to-noise ratio enhances acoustic sampling of wildlife
}

\author{
Kevin FA Darras ${ }^{\text {Corresp., }}{ }^{1}$, Franziska Deppe ${ }^{1}$, Yvonne Fabian ${ }^{1}$, Agus P Kartono ${ }^{2}$, Andres Angulo ${ }^{1}$, Bjørn Kolbrek ${ }^{3}$, \\ Yeni A Mulyani ${ }^{2}$, Dewi M Prawiradilaga ${ }^{4}$ \\ ${ }^{1}$ Agroecology, University of Göttingen, Göttingen, Niedersachsen, Germany \\ Department of Forest Resources, Conservation and Ecotourism, Bogor Institute of Agriculture, Bogor, Indonesia \\ 3 Celestion, Ipswich, Suffolk, United Kingdom \\ ${ }^{4}$ Cibinong Science Centre, Research Centre for Biology LIPI, Bogor, Indonesia \\ Corresponding Author: Kevin FA Darras \\ Email address: kdarras@gwdg.de
}

Background. Automated sound recorders are a popular sampling tool in ecology. However, the microphones themselves received little attention so far, and specifications that determine the recordings' sound quality are seldom mentioned. Here, we demonstrate the importance of microphone signal-tonoise ratio for sampling sonant animals.

Methods. We tested 12 different microphone models in the field and measured their signal-to-noise ratios and detection ranges. We also measured the vocalisation activity of birds and bats that they recorded, the bird species richness, the bat call types richness, as well as the performance of automated detection of bird and bat calls. We tested the relationship of each one of these measures with signal-tonoise ratio in statistical models.

Results. Microphone signal-to-noise ratio positively affects the sound detection space areas, which increased by a factor of 1.7 for audible sound, and 10 for ultrasound, from the lowest to the highest signal-to-noise ratio microphone. Consequently, the sampled vocalisation activity increased by a factor of 1.6 for birds, and 9.7 for bats. Correspondingly, the species pool of birds and bats could not be completely detected by the microphones with lower signal-to-noise ratio. The performance of automated detection of bird and bat calls, as measured by its precision and recall, increased significantly with microphone signal-to-noise ratio.

Discussion. Microphone signal-to-noise ratio is a crucial characteristic of a sound recording system, positively affecting the acoustic sampling performance of birds and bats. It should be maximised by choosing appropriate microphones, and be quantified independently, especially in the ultrasound range. 


\section{High microphone signal-to-noise ratio 2 enhances acoustic sampling of wildlife}

3

\section{Authors}

5 Kevin Darras ${ }^{1}$, Franziska Deppe ${ }^{1}$, Yvonne Fabian ${ }^{1}$, Agus Priyono Kartono ${ }^{2}$, Andres Angulo ${ }^{1}$, 6 Bjørn Kolbrek ${ }^{3}$, Yeni A. Mulyani ${ }^{3}$, Dewi M. Prawiradilaga ${ }^{4}$

7

$8{ }^{1}$ Agroecology, Department of Crop Sciences, University of Göttingen

$9{ }^{2}$ Wildlife Ecology, Department of Forest Resources, Conservation and Ecotourism, Faculty of

10 Forestry, IPB University

$11{ }^{3}$ Celestion, Ipswich, Suffolk, IP6 0NL, UK

$12{ }^{4}$ Research Centre for Biology LIPI, Cibinong Science Centre, Jalan Raya Jakarta Bogor KM 46, 13 Bogor 16911, Indonesia

14

15 Corresponding author:

16 Kevin Darras

17 Agroecology, Grisebachstrasse 6, Department of Crop Sciences, University of Göttingen, 37077

18

19

20

21
Göttingen, Germany

kdarras@gwdg.de 


\section{Abstract}

23 Background. Automated sound recorders are a popular sampling tool in ecology. However, the microphones themselves received little attention so far, and specifications that determine the

25 recordings' sound quality are seldom mentioned. Here, we demonstrate the importance of microphone signal-to-noise ratio for sampling sonant animals.

Methods. We tested 12 different microphone models in the field and measured their signal-tonoise ratios and detection ranges. We also measured the vocalisation activity of birds and bats that they recorded, the bird species richness, the bat call types richness, as well as the performance of automated detection of bird and bat calls. We tested the relationship of each one of these measures with signal-to-noise ratio in statistical models.

Results. Microphone signal-to-noise ratio positively affects the sound detection space areas, which increased by a factor of 1.7 for audible sound, and 10 for ultrasound, from the lowest to the highest signal-to-noise ratio microphone. Consequently, the sampled vocalisation activity increased by a factor of 1.6 for birds, and 9.7 for bats. Correspondingly, the species pool of birds and bats could not be completely detected by the microphones with lower signal-to-noise ratio. The performance of automated detection of bird and bat calls, as measured by its precision and recall, increased significantly with microphone signal-to-noise ratio.

Discussion. Microphone signal-to-noise ratio is a crucial characteristic of a sound recording system, positively affecting the acoustic sampling performance of birds and bats. It should be maximised by choosing appropriate microphones, and be quantified independently, especially in the ultrasound range. 


\section{Introduction}

47 Acoustic recording of wildlife is a popular sampling method for sonant animals such as birds and

48 bats (Gibb et al., 2018; Sugai et al., 2019). The ecologist can control the sound recording quality

49 by choosing high-quality microphones, ensuring that the animal sounds of interest can be

50 detected (Fristrup \& Mennitt, 2012). Microphone quality is commonly described by its self-noise

51 and signal-to-noise ratio (SNR). Self-noise is the noise produced by the microphone in the

52 absence of sound, and is typically given in dB SPL (decibel sound pressure level, defined as 20

53 times the logarithm of the ratio of the sound pressure to the reference sound pressure of $20 \mu \mathrm{Pa}$ )

54 A-weighted. It describes the equivalent background noise level that would be measured by a

55 perfect (noiseless) microphone, and is ideally measured by placing the microphone in a sound-

56 proof container. Microphone self-noise defines the lowest sound pressure level the microphone

57 can detect, and also the resulting SNR of the recorded signals. Signal-to-noise ratio in dB is

58 defined as 10 times the logarithm of ratio of a standard signal's power to the noise power of the

59 microphone created by its self-noise (Kinsler et al., 1999; International Organization for

60 Standardization, 2019):

$61 S N R=10 \times\left(\frac{\text { Power }_{\text {signal }}}{\text { Power }_{\text {noise }}}\right)$

62 The standard signal is commonly generated by a sound calibrator with a $94 \mathrm{~dB}$ SPL tone at a 1

$63 \mathrm{kHz}$ sound frequency. Signal-to-noise ratio is a relative measure, valid only for a given signal

64 level, while self-noise is an absolute measure of the microphone quality. Signal-to-noise ratio at

65 a calibrated SPL will however give a measure of self-noise, because it is obtained by subtracting

66 the self-noise from the standard signal's level.

67 In the following, we will focus on the more commonly mentioned SNR rather than the 
68 microphone self-noise. Its importance is routinely implied in technical literature about

69 microphones (e.g., Lewis \& Schreier, 2013). In contrast, in ecoacoustics, little attention has been

70 paid to the microphone specifications. Some studies have evaluated the effectiveness of different

71 recorder types for birds (Rempel et al., 2013; Pérez-Granados et al., 2019) and bats (Adams et

72 al., 2012), and it has been reported that a recording system with the lowest SNR detected the

73 least birds (Rempel et al., 2013). Finally, Bardeli et al. (2010) mentioned that automated

74 detection of animal sounds could be impeded by worn microphones. However, out of 20

75 published studies used in a recent meta-analysis about autonomous sound recording (Darras et

76 al., 2018a), only six mentioned SNR, and only two of those specified the SNR of their own

77 microphones. However, it is the first element in the signal processing chain and it determines the

78 output recording's quality.

79 Technical specifications of microphones, including their SNR, have an impact on the sampling

80 effectiveness, probably through their impact on the detection ranges: microphones that have a

81 low SNR (a high self-noise) add too much noise to the recordings, so that the animal sounds -

82 especially faint, distant ones - are not detectable anymore (Darras et al., 2018a). However, it is

83 crucial to maximise detection ranges to cover the largest possible sampling areas in little time. So

84 far, an experimental proof of the relationship between SNR and detection ranges is still missing,

85 although SNR is a technical measure that we can control by design, in contrast to the other biotic

86 and abiotic factors that determine sound detection spaces (Darras et al., 2016). Moreover, since

87 high SNR leads to less noise in recordings, the recordings would be easier to analyse for human

88 listeners. By extension, high SNR also facilitates the automated detection of animal sounds

89 (Jančovič \& Köküer, 2011), as well as their classification (Chen \& Maher, 2006), both of which

90 are commonly used in acoustic bat surveys, and increasingly used for bird surveys 
91 (Priyadarshani, Marsland \& Castro, 2018).

92 We aim to understand how microphone SNR affects the recording of soundscapes for ecologists

93 sampling sonant biodiversity. We used 12 different microphone models spanning a wide range of

94 SNR values to evaluate how it affects acoustic sampling of birds and bats, the most frequently

95 acoustically sampled animals. In the field, we recorded silence to determine the self-noise floor

96 of the microphones, sound transmission sequences to determine the microphones' SNR and their

97 detection ranges, and bats at night and birds during the morning to determine their sampling

98 efficiency. We measured the microphones' calibrated SNR values and compared them to

99 manufacturer specifications to check their reliability; for the first time, we measured microphone

100 SNR in the ultrasound range. We tested whether SNR determines the detection ranges of the

101 microphones at audible and ultrasonic frequencies. Since detection ranges determine the acoustic

102 sampling areas, we ultimately tested whether SNR affects the measured activity and richness of

103 the sampled birds and bats. Additionally, we tested the relationship between SNR and the

104 precision and recall of and bird and bat call automated detection. 
105 Materials and methods

\section{Microphones}

107 We used omnidirectional microphone elements of different types and models to test a wide range

108 of manufacturer-specified SNR values that are typical for soundscape recording applications. We

109 used two units for each of 12 different models from six different manufacturers, with specified

110 SNR at $1 \mathrm{kHz}$ from 55 to $80 \mathrm{~dB}$, resulting in a total of 24 microphone elements (Table 1). Most

111 of the chosen microphones are used in commercial or open-source microphones for soundscape

112 recording. Eight microphone element models were in the form of traditional cylindrical capsules,

113 and two of them are used in commercial microphones (WM-61A in SMX-II, FG-23629-C36 in

114 SMX-U1, Wildlife acoustics). The AOM-5024L-HD-R is interchangeable with the EM172

115 element used in the Solo open-source recording system (Whytock \& Christie, 2017). Four

116 models were Micro-Electro-Mechanical Systems (hereafter MEMS) chips that can be integrated

117 on printed circuit boards, two of which were used in commercial products (SPU0410LR5H-QB

118 in Bio-SMX-US, Biotope.fr, SPM0404UD5 in SMX-US, Wildlife acoustics). Four of the MEMS

119 elements and one capsule microphone element are also part of the open-source microphone

120 system for ecoacoustics "Sonitor" (Darras et al., 2018b). Finally, to extend the range of tested

121 SNR values, we included a microphone with a lower SNR (POM-1345P-C3310-R, $55 \mathrm{~dB}$ ) that is

122 not used in microphones for soundscape recording. The prices per unit at purchase ranged from

123 approximately 0.5 EUR (typical for MEMS elements) to 4 EUR (typical for high-end capsules),

124 depending on the ordered quantity, with the notable exception of the FG Knowles element,

125 which cost 23 EUR. Details about microphone assembly are in the supplementary materials.

126 Our microphones were calibrated at 1 and $40 \mathrm{kHz}$ using reference microphones for audible sound 
127 calibration, because not all had the same, standard format to fit a sound calibrator. For audible

128 sound, we used the SMX-US (discontinued - Wildlife Acoustics, Massachusetts, USA): it has a

129 standard 1/2 inch diameter, fitting into a class I sound calibrator (PCE-SC42, PCE instruments,

130 Germany) that emits a $94 \mathrm{~dB}$ SPL tone of $1 \mathrm{kHz}$. For ultrasound, we used the reference

131 microphone ICS-40720; it was calibrated with the ultrasound calibrator (Wildlife Acoustics,

132 Massachusetts, USA) that emits a $48 \mathrm{~dB}$ SPL tone of $40 \mathrm{kHz}$ in "chirp" mode (measured at a

133 distance of $30 \mathrm{~cm}$, because microphones were not plugged into it).

\section{Study site and setup}

135 We set up the microphones in a research plot situated in an oil palm plantation (S01.70725,

136 E103.39781, WGS84 datum) belonging to the PTPN6 state company in Sumatra, Indonesia._Our

137 research group was part of the CRC990 project, which has a Memorandum of Understanding

138 with PTPN6. We installed 12 microphones - one of each model - simultaneously in a microphone

139 holder, to equalise the soundscapes that they record. The holder consisted of a wooden pane (Fig

$140 \mathrm{~S} 1$, approximately $25 \mathrm{~cm} \times 35 \mathrm{~cm}$, installed at a height of $1.5 \mathrm{~m}$ ) fitted with synthetic foam strips

141 to absorb sound reflections. The holes where the microphones were inserted were also padded

142 with foam to avoid friction noise, and positioned horizontally. The microphones were arranged

143 in a regular grid, with 3 microphones in each of 4 rows, spaced $10 \mathrm{~cm}$ vertically and $15 \mathrm{~cm}$

144 horizontally. We removed wind screens, which incur a slight loss in sound transmission $(<1 \mathrm{~dB}$

145 according to Wildlife Acoustics), to measure the performance of the actual microphone elements.

146 We recorded on rain- and wind-free days, as most microphones were not protected. The

147 microphones were connected to six sound recorders (SM2Bat+, Wildlife Acoustics) with $5 \mathrm{~m}$

148 cables. We chose the SM2Bat+ because it was the only recorder that we possessed which was

149 compatible with all microphones. The manufacturer specifies an electric noise floor of $-115 \mathrm{~dB}$ 
$150 \mathrm{~V}$ for $44.1 \mathrm{kHz}$ recordings and $-105 \mathrm{~dB} \mathrm{~V}$ for $192 \mathrm{kHz}$ recordings. After completing all

151 measurements with the first set of microphones, we repeated them with the second set on the

152 following day, placing the second unit of each model in the same position as with the previous

153 set.

\section{Sound level measurement}

155 We recorded all sound in uncompressed audio (WAV format) in stereo (2 channels), sampled at

$156192 \mathrm{kHz}$ (16 bit per sample) in November 2018. We visualized, processed, and measured audio

157 in Audacity (Audacity Team, 2018). We generated their spectrograms with a Fast-Fourier-

158 Transform with a Hanning window size of 1024. For measuring sound levels, we re-sampled

159 audio at a sampling rate of $96 \mathrm{kHz}$ to obtain a higher frequency resolution in the spectrograms.

160 We measured relative sound levels in $\mathrm{dB}$ in the frequency bins containing the $1 \mathrm{kHz}$ and at 40

$161 \mathrm{kHz}$ test frequencies using the "Plot spectrum" function. We used a $0.8 \mathrm{~s}$ sound selection for

162 audible signals and $0.07 \mathrm{~s}$ for the short ultrasound chirps. We chose to consistently use the 1024

163 window size as it is the default setting in Audacity that allowed the best trade-off between

164 temporal and frequency resolutions for locating and measuring the short $40 \mathrm{kHz}$ signal tone in

165 space and time. Since we are dealing with field measurements with noise outside of the test

166 frequencies, we did not use root-mean-squared values to measure sound pressure levels as they

167 would cover the entire frequency spectrum. Moreover, we needed to apply filtering in the

168 following for aurally and visually detecting those signals, justifying the use of sound levels

169 derived from specific frequency bins.

\section{Self-noise}

171 We measured microphone self-noise by recording sound in an environment that was as silent as

172 possible. We did not have access to an anechoic chamber and preferred to record silence in the 
173 oil palm plantation, far from anthropogenic machinery noise rather than in the laboratory or other

174 urban buildings. We used an isolating, large cylindrical ice box with a hole in its cover to pass

175 the microphone cables. The box was padded inside with synthetic foam and surrounded by a

176 thick polyester sleeping bag to prevent extraneous noise from reaching the microphones,

177 resulting in a basic anechoic box. We started the recording on all recorders, knocked on the box

178 to be able to synchronize them later, and recorded silence for one minute at a $96 \mathrm{kHz}$ sampling

179 rate. We measured the relative sound pressure levels in $\mathrm{dB}$ inside the silent recording for all

180 microphones at 1 and $40 \mathrm{kHz}$. We used the same simultaneous $60 \mathrm{~s}$ of sound for all microphones

181 of one set.

\section{Sound transmission sequences}

183 We recorded sound transmission sequences (Darras et al., 2016) to determine relative

184 microphone sensitivity, to compute SNR, and to measure detection spaces. We generated an

185 audio recording in Audacity, consisting of a sequence of $1 \mathrm{~s}$ long test tones at $1 \mathrm{kHz}$, repeated at

1865 different sound levels (see Supplementary materials) to be able to choose the most appropriate

187 sound level a posteriori. Audible test sounds came from a battery-powered one driver

188 loudspeaker (SoundCore Anker) at default loudness (the level automatically reset to when

189 powering on), with the audio recording loaded on a Mini-SD card. We emitted ultrasound with

190 the ultrasonic calibrator, which produces $40 \mathrm{kHz}$ chirps of constant loudness in "Chirp" mode.

191 Sound transmission sequences are obtained by recording test tones emitted at different distances

192 from the microphone: The loudspeaker and ultrasound calibrator were held at a height of $2 \mathrm{~m}$ and

193 pointed to the microphones as they emitted test sounds at distances of $2,4,6,8,10,15,20,25$,

$19430,35,40,45$, and $50 \mathrm{~m}$ to the front, the left, the right, and the back of the microphones to

195 determine the entire sound detection area. At 1 and $40 \mathrm{kHz}$, we used the nearest common 
196 distance at which none of the microphones recorded clipped (saturated) test tones to measure

197 their sensitivity relative to the ones recorded by the calibrated reference microphone; these tones

198 had the same source sound pressure level and distance to all microphones.

\section{Sound detection spaces}

200 For measuring the microphones' detection ranges, we chose the loudest of the five recorded

201 sound levels at which none of the microphones recorded detectable (i.e. not visible on

202 spectrogram and not audible in recording) test tones at $50 \mathrm{~m}$ by focusing on the relevant

203 frequency (1 or $40 \mathrm{kHz}$ ). By doing this, we ensured that we measured maximal detection ranges

204 for each microphone, and obtained the most accurate relative range differences. We determined

205 the detection range as extinction distances: the distance at which the test tone was not detectable

206 anymore because it equals the ambient sound level (Darras et al., 2016). Since above $10 \mathrm{~m}$, we

207 only emitted test tones every $5 \mathrm{~m}$, a single listener (FD) estimated the detection range to the

208 meter based on how loud the last detectable test sound was (visually assessed from the

209 spectrogram). For some combinations of microphone and direction however, detection ranges

210 exceeded $50 \mathrm{~m}$ at $1 \mathrm{kHz}$, so the listener estimated the extrapolated detection range above $50 \mathrm{~m}$.

211 The detection ranges in the four different directions formed four quarter-ellipses which were

212 used to calculate the sound detection space areas (using coord_polar function of ggplot2). We

213 did not use the mean detection ranges in our analysis because our setup could have resulted in

214 directional sound pickup patterns, and also because detection area is ultimately the measure that

215 determines the sampling area for our organisms of interest. The sound detection spaces we

216 measured represent standardised detection ranges that are specific to the particular sounds we

217 emitted; they are characterised by their frequency, the emitting hardware's source level,

218 directivity, height, orientation, and the habitat of the recording site (Darras et al., 2016). This is 
219 in contrast to effective detection radii: these depend on the organisms' vocalising frequency,

220 amplitude, orientation, and directivity, and they represent maximum distances up to which

221 specific species can be recorded with a particular sound recording system and habitat (Matsuoka

222 et al., 2012).

223

\section{Bird and bat activity and richness}

225 After carrying out the sound transmission recordings with each set, the microphones were left in

226 place to record sound on the following night (for bats) and morning (for birds). We used a

227 sampling rate of $44.1 \mathrm{kHz}$ during the day for birds and the usual $192 \mathrm{kHz}$ sampling rate for bats

228 at night. After retrieval, the recordings' sound levels were normalised (Normalize function in

229 Audacity).

230 We uploaded normalised recordings of the first 30 minutes after sunrise for birds and the first 30

231 minutes after sunset for bats to our online platform for ecoacoustics Biosounds

232 (https://soundefforts.uni-goettingen.de/biosounds/collection/show/31/4/gallery). We screened

233 synchronous recordings from every microphone, opened side-by-side in different internet

234 browser tabs. The recordings' spectrograms and audio enabled visual and aural filtering and

235 detection of bird and bat vocalisations by FD. The recordings that appeared to have the best

236 quality (clearest spectrogram and noise-free audio) were used as reference (Knowles FG-23629-

237 C36 for bats, Primo EM258 for birds) as clear differences were visible in the spectrograms

238 between microphones (Fig S2). Whenever a vocalisation was found in the reference recording, it

239 was annotated to extract its coordinates in time. Calls of the same species that were separated by

240 less than 15 (for birds) or 5 seconds (for bats) were put in a common tag to ensure standardised

241 tagging among recordings; tagging every call is too tedious and does not reflect the activity of 
242 birds singing at large intervals accurately. Note that different thresholds could result in slightly

243 different results and that different tags could belong to different or identical individuals.

244 However, it is more important to use a consistent threshold across the variables of interest (here,

245 the microphones) for unbiased results. All other non-reference recordings were checked at the

246 same time point and if vocalisations could be found, they were also tagged. We checked whether

247 other vocalisations were missed in the non-reference recordings that could not be found in the

248 reference recording.

249 We measured bird and bat activity and richness. AA manually assigned bird vocalisations to

250 species using Birdlife International taxonomy, and FD manually assigned bat vocalisations to

251 call types due to the lack of comprehensive reference libraries for South-East Asia, and also

252 because bat species identity per se does not affect our results. We counted richness as the

253 number of bird species or bat call types for each microphone. We computed the total duration of

254 tagged vocalisations for birds and bats for each microphone, which yielded the vocalisation

255 activity, in seconds.

\section{Automated detection of calls}

257 We carried out automated detection of bird and bat calls with "acoustic recognizers". We 258 exported the same, loudest bird and bat call sequence (to ensure that they are recorded by all 259 microphones) of the most common species from each microphone's unamplified recording. 260 Along with these call sequences, we exported two representative calls contained within them,

261 which were also the same across microphones. We used the latter as templates to detect all other

262 calls in the exported bird and bat call sequences using spectrogram cross-correlation with

263 monitoR (Katz, Hafner, \& Donovan, 2016). We ordered the detections made by monitoR by

264 descending score and AA manually assigned them to true and false positives by visually 
265 checking their spectrograms. After twenty consecutive false positives, we deemed all the

266 following detections to be false positives also. We counted the actual number of calls (equal to

267 the sum of true positives and false negatives) visually in the spectrogram of the bird and bat call

268 sequences. Bat calls from the second night were distant, so that they were not picked up by three

269 microphones and too rare for the other microphones.

\section{Statistical analysis and graphing}

271 Statistical tests were performed in R 3.6.1 (R Core Team, 2018); plots were drawn using ggplot2

272 (Wickham, 2009); the code and output can be checked in the HTML report (Supplementary

273 Materials). Actual effect sizes for the detection area and vocalisation activity models are hard to

274 interpret due to different predictor transformations and the use of different generalised model

275 families; they are also specific to our study site, so we report the ranges of the response variables 276 instead.

277 Measured vs. specified signal-to-noise

278 The relative sound pressure levels output by the recorder were standardised by subtracting the

279 amplification applied by the recorder. We then computed the SNR of each microphone by

280 subtracting its relative sensitivity difference and its self-noise from the sound pressure level of

281 the calibration tone recorded by the reference microphone. The obtained SNR is relative to the

282 absolute sound pressure level of the calibrators ( $94 \mathrm{~dB} \mathrm{SPL}$ for $1 \mathrm{kHz}, 48 \mathrm{~dB}$ SPL for $40 \mathrm{kHz}$ ).

283 We plotted the measured and specified (i.e. manufacturer given) microphone SNR to check how

284 consistent they are across manufacturers. Note that we did not expect our SNR values to equal

285 manufacturer specifications due to the different procedures used for measuring them: we use

286 frequency-specific measures of sound level while manufacturers use broad-band root-means-

287 squared measures of sound level. However, we expected that similar specified SNR values 
288 across microphones of different manufacturers yield similar measured SNR values. Some

289 microphones had acoustic vents (GAW112, Gore, USA) in front of them, which reduce sound

290 transmission by $<1 \mathrm{~dB}$ (specified by the manufacturer) while providing protection against water

291 ingress; we corrected this by adding $1 \mathrm{~dB}$ to their measured SNR, only when comparing them to

292 manufacturer SNRs. We compared the corrected Akaike Information Criterion (Sugiura 1978) of

293 linear models predicting the measured SNR using all combinations of the continuous specified

294 SNR and the categorical manufacturer predictor: the null model without predictors, a simple

295 model with specified SNR only, one with an additional manufacturer-specific offset, and one

296 with the additional interaction between manufacturer and specified SNR. We used the model

297 with the lowest AICc - and consequently highest predictive power - to draw regression lines of

298 predicted values of measured and specified SNR and checked the plot of its simulated residuals

299 (DHARMa package in R).

300

301

302

303

304

305

306

307

308

309

310

\section{Signal-to-noise vs. detection space area}

We tested for the relationship between microphone SNR and sound detection space area, depending on the frequency, using a linear regression model. Depending on the range of SNR values under consideration, SNR may have an accelerating effect on detection spaces at low SNR values (a linearly increasing range leads to a quadratic increase in the detection circle's area), a roughly linear effect at intermediate SNR values, and a decelerating effect at high SNR values, where the detection area is increasingly limited by the ambient sound (Darras et al., 2018a). As a consequence, we tested three models: one with a linear SNR predictor, one with a second-order polynomial of the SNR predictor (for modeling convex and concave relationships), and one with a log-transformed SNR predictor (which has one more degree of freedom than the second-order polynomial, but cannot model an increasing slope). We compared the AICc value 
311 of our models to choose the one with the highest predictive power and checked the plot of its

312 simulated residuals. Note that we did not model more complex relationships between SNR and

313 detection area because a multitude of interacting, and sometimes opposed factors affect sound

314 transmission (Darras et al., 2016).

315 Signal-to-noise vs. vocalization activity

316 We tested the relationship between microphone SNR and bird and bat activity using a model

317 with the sampling day as an additional fixed effect to account for day-to-day variations in animal

318 activity. We chose the best SNR predictor transformation as determined by the analysis of

319 detection spaces, corresponding to the frequency of interest. For the bird and bat activity

320 datasets, we tested different model types to obtain the best fit. We tested a linear model that

321 accounts for the fact that at high values, the activity distribution can tend to normality (Central

322 limit theorem); we tested a generalised model with poisson family to account for the fact that

323 activities are derived from a count of animals; we tested a model with a negative-binomial family

324 to account for overdispersion, and finally, we tested a model with Gamma family to account for

325 the fact that activities are positive and continuous values. We compared the AICc value of our

326 models to choose the one with the highest predictive power and checked the plot of its simulated

327 residuals.

\section{Signal-to-noise vs. species richness}

329 The sampled species richness can be affected by microphone SNR either by the speed at which

330 the maximum number of sampled species is reached, or by the maximum level of sampled

331 species itself. We counted the number of sampled species at 40 time steps along the 30 minutes

332 of recording, to graphically depict the species pool and the speed at which it is sampled with time

333 for microphones with different SNR. We refrained from analysing the effect of SNR on species 
334 richness with statistical models for the following reasons: 1) oil palm plantations have limited

335 species pools: in our study area, we expect no more than four call types for echolocating bats,

336 and seven bird species (Darras et al., 2019), so that we only have a very limited range for the

337 response variable; 2) typical count-variable models (such as poisson or negative binomial) are

338 modeling unbounded count response variables, and as such inappropriate; 3) the statistical

339 analysis of the effect of SNR could be carried out at each of the different time steps at which the

340 species richness is counted, but count-variable models would not converge at early time steps

341 when the response variable comprises mostly zeroes, at intermediate time steps, the results are

342 driven by outliers, and at late time steps, we are essentially only testing the effect of SNRs on the

343 maximal level of the species pool only, which is very limited; 4) modeling the species

344 accumulation with time using saturating curves with a Michaelis-Menten dynamic yields too

345 many unrealistic outliers that do not correspond to the maximal number of species present in oil

346 palm plantations.

\section{Signal-to-noise vs. automated detection performance}

348 We analysed the influence of the microphone SNR on the performance of the automated

349 detection algorithm used in monitoR, which matches a template with each time bin in the

350 recording (Katz, Hafner \& Donovan, 2016). We follow the recommendations of Knight et al.,

351 (2017) to assess the performance of acoustic automated detection algorithms (i.e., acoustic

352 recognizers) with the precision - which describes the proportion of detections that are true

353 positives - and the recall - which describes the proportion of the real number of calls that are

354 detected by the recognizer. In line with their recommendations, we account for the influence of

355 the threshold (or cutoff) of the score, which measures the level of confidence in the detection,

356 used for classifying audio events as detections or not, which ranges from 0 (no match with 
357 template) to 1 (perfect match). We depicted the total number of available calls along with the

358 true and false positives, in relation to the score cutoff used to count them. We defined minimal

359 precision and recall levels of 0.5 as acceptable and computed the range of score cutoff values

360 over which we would obtain an acceptable automated detection performance. Finally, we

361 analysed the effect of SNR on the acceptable range of score cutoffs with a linear model, using

362 the sampling day and frequency as fixed effects, along with the interaction between SNR and

363 frequency. We checked the simulated residual plots with DHARMa and assessed the effect size

364 and P-value of the SNR predictor for both frequencies. 


\section{Results}

\section{Measured vs. specified signal-to-noise}

367 The linear model using specified SNR and manufacturer-specific intercepts as predictors had the

368 highest predictive power (i.e. lowest AICc: Table 2; Fig 1) and the simulated residuals showed

369 no deviations (HTML report, supplementary materials). We detected large differences between

370 manufacturers: for instance, we measured SNR values for PUI Audio that were on average $26 \mathrm{~dB}$

371 below those we measured for Knowles microphone elements.

372 Signal-to-noise vs. detection space area

373 The model with log-transformed SNR that predicted detection areas at $1 \mathrm{kHz}$ had the lowest

374 AICc, while the model with non-transformed SNR that predicted detection areas at $40 \mathrm{kHz}$ had

375 the lowest AICc (Table 3). The simulated residuals of the chosen models showed no deviations

376 (HTML report, supplementary materials). Detection areas increased significantly with SNR from

377207 to $10477 \mathrm{~m}^{2}$ at $1 \mathrm{kHz}$ and from 353 to $3632 \mathrm{~m}^{2}$ at $40 \mathrm{kHz}$ (all $\mathrm{P}<0.001$, adjusted $\mathrm{R}^{2}$ at 1

378 kHz: 0.91; at $40 \mathrm{kHz}$ : 0.70; Fig. 2). Detection ranges roughly corresponded to beams of omni-

379 directional microphones (Fig S3).

\section{Signal-to-noise vs. vocalisation activity}

381 We detected nine bird species (Chalcophaps indica, Dicaeum trigonostigma, Geopelia striata,

382 Halcyon smyrnensis, Orthotomus atrogularis, Orthotomus ruficeps, O. sericeus, Pycnonotus

383 aurigaster, $P$. goiavier) and four bat call types (A-D). The linear model that predicted bird

384 activities with log-transformed SNR had the lowest AICc, and the generalised negative binomial

385 linear model that predicted bat activities with SNR had the lowest AICc (Table 4). The simulated 
386 residuals of the chosen models showed no strong deviations (HTML report, supplementary

387 materials). The sampled vocalisation activities significantly increased with SNR (Fig. 3). During

388 the day with most animal activity, activities ranged from 950 to $1539 \mathrm{~s}$ for birds ( $\mathrm{P}<0.001$,

389 adjusted $\mathrm{R}^{2}$ : 0.92 , using only microphones commonly employed for ecoacoustics), and from 33

390 to $320 \mathrm{~s}$ for bats $\left(\mathrm{P}<0.001\right.$, adjusted $\left.\mathrm{R}^{2}: 0.90\right)$. The microphone model with the lowest $\mathrm{SNR}$

391 (POM-1345P-C3310-R) did not pick up any bird calls.

392 Signal-to-noise vs. species richness

393 Maximal bat species richness levels generally reached higher levels, at a higher rate, with

394 increasing microphone SNR (Fig. 4). For birds, we could only observe higher maximal sampled

395 richness levels with SNR for one of the two sampling days ( $5^{\text {th }}$ of November).

396 Signal-to-noise vs. automated detection performance

397 The performance of automated detection of bird and bat calls was positively affected by

398 microphone SNR (Fig. 5). The simulated residuals showed no deviations (HTML report,

399 supplementary materials). The range of score cutoff values that had acceptable recall and

400 precision (i.e., a minimum of 0.5 ) significantly increased by 0.9 and 0.4 per SNR unit increase,

401 respectively for bird and bat calls (all $\mathrm{P}>0.001$, adjusted $\mathrm{R}^{2}: 0.80$ ). 


\section{Discussion}

403 Our measured SNR values showed large discrepancies between microphone element

404 manufacturers. Increasing signal-to-noise ratios considerably enlarged sound detection areas for

405 audible sound and ultrasound. In turn, the sampled bird and bat activity and richness was largely

406 enhanced by high microphone SNR, and automated detection performance of bat calls was

407 facilitated by high microphone SNR.

\section{Measuring signal-to-noise ratios and detection ranges}

409 For ecoacoustic studies, we need standardised microphone SNR values for specific frequencies

410 of interest corresponding to different animal groups. Although we only measured two

411 frequencies, SNR is a continuous measure that varies with frequency, and depending on the

412 targeted organism, other frequencies (or frequency bands) will be more relevant to determine the

413 fit of different microphones. Microphone manufacturer-provided SNR did not correlate well with

414 our standardised SNR measurements because of strong differences between manufacturers.

415 Microphone element manufacturers do not follow a standard certification for measuring

416 microphone SNR (pers. comm. with Vesper and PUI audio representatives). Moreover, SNR

417 values are usually only specified for $1 \mathrm{kHz}$, a representative frequency for human speech (but see

418 Knowles, 2014), so that we could not compare our signal-to-noise measurements at $40 \mathrm{kHz}$ with

419 any reference. However, for bat researchers, we showed that it is important to have high-SNR

420 microphones, but this cannot be achieved without knowing their ultrasonic SNR beforehand.

421 Furthermore, SNR values at $1 \mathrm{kHz}$ are only weakly indicative of microphone performance in the

422 ultrasound range (Fig S4), even though some of the variation in our ultrasound SNR values

423 might have been caused by variable alignment of the microphones. Finally, our self-noise 
424 measurements were carried out for specific frequencies, but manufacturers usually measure

425 signal-to-noise - which is based on the self-noise measure - with A-weighting (which weights

426 human-audible frequencies more) over a $20 \mathrm{~Hz}$ to $20 \mathrm{kHz}$ bandwidth that encompasses different

427 groups of vocalising animals. Moreover, different weightings and bandwidths can lead to

428 different SNR values. In future studies, SNR measurements in audible sound and ultrasound

429 ranges should be compiled by researchers with standard measurement protocols for different

430 microphone models to support microphone selection for ecoacoustic studies. Note that as SNR

431 varies with frequency, it can be derived from the frequency and noise responses of the

432 microphones to frequency by subtraction, for a more complete assessment of microphone quality

433 across the entire acoustic frequency range. Unfortunately, such data are rarely available (but see

434 Knowles, 2014).

435 Using our sound detection area measurement approach, one could also directly benchmark

436 microphones based on their detection ranges. Even though signal-to-noise ratio seems to be the

437 main determinant of sound detection spaces (and is more easily measured), other microphone

438 characteristics like linearity and directionality theoretically also affect them. Even though signal-

439 to-noise ratio seems to be the main determinant of sound detection spaces (and is more easily

440 measured), other microphone characteristics like linearity and directionality theoretically also

441 affect them. However, all the microphones we used were specified as linear and omnidirectional

442 by the manufacturers, and any possible deviation from this was assumed to be random across

443 microphone models. It would be possible to devise protocols to measure the sound detection

444 spaces of sound recording setups - recorders with microphones, or only microphones - of

445 different manufacturers to assess their sampling effectiveness in a standardised way. Relative

446 differences should be independent of the habitat in which recordings are made, but absolute 
447 ranges would vary between habitats (Darras et al., 2016). Possibly, results of previously

448 published comparisons of bird and bat recorders (Adams et al., 2012; Rempel et al., 2013), which

449 did not explicitly consider microphones, could be explained quantitatively by simple differences

450 in detection ranges caused by differing microphone SNR values.

451 We consider that by definition, sensitivity is secondary compared to self-noise or SNR values

452 with respect to their impact on detection ranges, in slight contrast to previous findings about the

453 importance of microphone sensitivity (Turgeon, Van Wilgenburg \& Drake, 2017). Microphones

454 used for wildlife recordings usually have a sensitivity of $-36 \mathrm{dBV}$, their levels are equalised with

455 amplification, and the added noise that arises from this amplification is generally negligible:

456 Even with our discontinued recorders, and only at ultrasound sampling frequencies, only the

457 ICS-40720 and Primo EM258 microphone elements are noticeably limited by the recorder by

458 having higher electrical noise floors than the amplifier. Thus, we argue that for a broad range of

459 commercially available microphone elements that come into question for wildlife recordings,

460 SNR values are the limiting factor determining sound detection areas.

461 Maximising recording quality

462 The inherent sampling effectiveness of sound recorders, in terms of sampling area, should be

463 maximised by choosing microphones with high SNR values. In our case, we could reach half of

464 the largest detection area with SNRs as low as $42 \mathrm{~dB}$ for birds and $81 \mathrm{~dB}$ for bats. However, this

465 number depends on the range of SNR values covered by our selected microphones. In contrast, a

466 previous meta-analytical approach showed that microphones for audible sound perform as well

467 as human observers at SNR values of approximately $80 \mathrm{~dB}$ (Darras et al., $2018 \mathrm{a}$ ). Thus, more

468 importantly, detectability increases with SNR: even though extremely low SNR microphone

469 elements are almost non-existent, it is worthwhile to search for the highest SNR in the market of 
470 existing microphone elements. Moreover, the detection areas of our wide range of microphone

471 elements did not reach a clear saturation point, although detection spaces are eventually limited

472 by the ambient sound (Apol et al., 2018, Darras et al., 2018 a). Crucially, the best-performance

473 microphones can be obtained at little additional expense: as an example, the lowest signal-to-

474 noise PUI Audio microphone element (55 dB SPL) cost us 3.10 EUR (at the time of purchase,

475 via Digikey.com), only a little less (28 \%) than the highest signal-to-noise PUI Audio

476 microphone element ( $80 \mathrm{~dB}$ SPL) that cost 4.25 EUR. Compared to the total cost of

477 microphones, these expenses are negligible, and even more so with even cheaper MEMS

478 microphones, which can cost roughly half as much.

479 Low signal-to-noise microphones are not unusable, but they come with manageable drawbacks.

480 Even though it is more efficient to sample birds and bats with high-quality ratio microphones, in

481 some special cases where the sampling area should not be too large (e.g., when sampling needs

482 to be limited to small habitat patches), having small microphone detection ranges can be an

483 advantage. However, artificially adding noise to high-quality recordings could be a more flexible

484 approach and have the same effect (Darras et al., 2016). Also, sub-optimal recordings from low-

485 quality microphones can be used too, as long as longer recording durations are used, to measure

486 additively higher vocalisation activities and to reach higher species richness as shown by our

487 species accumulation curves (Fig. 3). Low-quality microphone recordings can also be used

488 together with high-quality recordings when accounting explicitly for the different detectabilities

489 (e.g., with occupancy modeling approaches), or for the relationship between vocal activity rate

490 and bird abundance (Pérez-Granados et al., 2019).

491 For species richness, the outcome of increasing SNR and sampling durations may depend on the

492 animals' mobility. For mobile organisms like bats that are constantly roaming the study site, the 
493 entire species pool could be sampled eventually - even when detection spaces are small because

494 of low-quality microphones - given enough sampling time. For territorial organisms like birds,

495 that have perching preferences and mostly vocalize when perching, the species pool could

496 remain incompletely sampled even after long recording durations. However, our results

497 contradicted our expectation, and we recommend more in-depth studies in habitats with larger

498 species pools, sampling for longer durations.

499 Still, high SNR values are required for accurate localisation of sound sources (Good \& Gilkey,

500 1996), which would support the estimation of bird detection distances for distance sampling

501 (Darras et al., 2018 b). In practice, recordings from microphones with high SNR also show

502 clearer signals with higher SNR (Fig S5). We showed how this facilitates automated detection:

503 microphones with low SNR do not have an acceptable performance in terms of precision and

504 recall. Analogously to sound recordings, the lower the SNR, the lower the signal of detection

505 events and the higher the signal of the background noise, to the point that it is impossible to

506 distinguish true detection events from spurious detection events found by the recognizer inside

507 ambient noise, leading to low precision. Correspondingly, the lower the SNR, the narrower the

508 range of score thresholds wherein automated detection performs satisfyingly, and in the worst

509 case, automated detection becomes impossible. It is also conceivable that analogously, high SNR

510 values facilitate visual and aural inspection of sound recordings by humans.

511 Finally, for all microphones, sensitivity and SNR values degrade with time, so that they should

512 be regularly assessed to keep sampling effectiveness to a maximum or to account for their

513 variable detection ranges (Darras et al., 2018 c, Turgeon et al., 2017). However, even with a

514 technically high-quality sound recording setup, the deployment should still follow general

515 recommendations for soundscape recordings (Darras et al., 2018a), such as installing 
516 microphones above the lower vegetation layer, spacing them and pointing them in opposite

517 directions, and using lossless compressed audio at high sampling rates.

\section{Conclusions}

519 We suggest that ecologists recognize microphone SNR or self-noise as a standard metric for 520 assessing microphone quality in ecoacoustics. Microphone SNR largely determines the sound

521 detection space. Through this, it dictates how many individuals are recorded and how efficiently

522 species pools can be sampled. High microphone SNR values also enhance the precision and

523 recall of automatic detection methods for bird and bat calls. Thus, high-quality microphones are

524 paramount for achieving maximum detection ranges with accurately detectable sounds.

\section{Acknowledgements}

526 We declare that the authors have no conflicts of interest. We would like to offer our most sincere

527 condolences to the family of Agus Priyono Kartono, who deceased during the study. We thank

528 the reviewers for their extremely valuable help in improving this paper. We are grateful to our

529 counterpart Damayanti Buchori for supporting this research project.

\section{Conflict of Interest}

533 The authors declare that they have no conflict of interest. 


\section{Data availability}

535 All data required to reproduce the results and figures will be provided in Dryad upon acceptance. 


\section{References}

Adams AM, Jantzen MK, Hamilton RM, Fenton MB. 2012. Do you hear what I hear? Implications of detector selection for acoustic monitoring of bats. Methods in Ecology and Evolution 3:992-998. DOI: 10.1111/j.2041-210X.2012.00244.x.

Bardeli R, Wolff D, Kurth F, Koch M, Tauchert K-H, Frommolt K-H. 2010. Detecting bird sounds in a complex acoustic environment and application to bioacoustic monitoring. Pattern Recognition Letters 31:1524-1534. DOI: 10.1016/j.patrec.2009.09.014.

Darras K, Batáry P, Furnas B, Celis-Murillo A, Wilgenburg SLV, Mulyani YA, Tscharntke T. 2018a. Comparing the sampling performance of sound recorders versus point counts in bird surveys: A meta-analysis. Journal of Applied Ecology 55:2575-2586. DOI: $10.1111 / 1365-2664.13229$.

Darras KFA, Corre MD, Formaglio G, Tjoa A, Potapov A, Brambach F, Sibhatu KT, Grass I, Rubiano AA, Buchori D, Drescher J, Fardiansah R, Hölscher D, Irawan B, Kneib T, Krashevska V, Krause A, Kreft H, Li K, Maraun M, Polle A, Ryadin AR, Rembold K, Stiegler C, Scheu S, Tarigan S, Valdés-Uribe A, Yadi S, Tscharntke T, Veldkamp E. 2019. Reducing Fertilizer and Avoiding Herbicides in Oil Palm Plantations-Ecological and Economic Valuations. Frontiers in Forests and Global Change 2. DOI: 10.3389/ffgc.2019.00065.

Darras K, Kolbrek B, Knorr A, Meyer V. 2018b. Assembling cheap, high-performance microphones for recording terrestrial wildlife: the Sonitor system. F1000Research 7:1984. DOI: 10.12688/f1000research.17511.1.

Darras K, Pütz P, Fahrurrozi, Rembold K, Tscharntke T. 2016. Measuring sound detection spaces for acoustic animal sampling and monitoring. Biological Conservation 201:29-37. DOI: 10.1016/j.biocon.2016.06.021.

Fristrup KM, Mennitt D. 2012. Bioacoustical monitoring in terrestrial environments. Acoustics 
Today 8:16-24.

Gibb R, Browning E, Glover-Kapfer P, Jones KE. 2018. Emerging opportunities and challenges for passive acoustics in ecological assessment and monitoring. Methods in Ecology and Evolution 10:169-185. DOI: 10.1111/2041-210X.13101.

International Organization for Standardization. 2019.ISO 80000-3:2019. Available at https://www.iso.org/cms/render/live/en/sites/isoorg/contents/data/standard/06/49/64974. html (accessed June 9, 2020).

Jančovič P, Köküer M. 2011. Automatic Detection and Recognition of Tonal Bird Sounds in Noisy Environments. EURASIP Journal on Advances in Signal Processing 2011:982936. DOI: $10.1155 / 2011 / 982936$.

Katz J. 2014. MonitoR: automation tools for landscape-scale acoustic monitoring. The University of Vermont.

Katz J, Hafner SD, Donovan T. 2016. Assessment of Error Rates in Acoustic Monitoring with the R package monitoR. Bioacoustics.

Kinsler LE, Frey AR, Coppens AB, Sanders JV. 1999. Fundamentals of Acoustics. New York: Wiley.

Knight E, Hannah K, Foley G, Scott C, Brigham R, Bayne E. 2017. Recommendations for acoustic recognizer performance assessment with application to five common automated signal recognition programs. Avian Conservation and Ecology 12. DOI: 10.5751/ACE01114-120214.

Knowles. 2014. Ultrasonic Applications for Knowles Electret and MEMS Microphones.

Pérez-Granados C, Bota G, Giralt D, Albarracín J, Traba J. 2019. Cost-Effectiveness Assessment of Five Audio Recording Systems for Wildlife Monitoring: Differences between Recording Distances and Singing Direction. Ardeola 66:311-325. DOI: 10.13157/arla.66.2.2019.ra4.

Pérez-Granados C, Bota G, Giralt D, Barrero A, Gómez-Catasús J, Rosa DB-DL, Traba J. 2019. Vocal activity rate index: a useful method to infer terrestrial bird abundance with acoustic 
monitoring. Ibis 161:901-907. DOI: 10.1111/ibi.12728.

Priyadarshani N, Marsland S, Castro I. 2018. Automated birdsong recognition in complex acoustic environments: a review. Journal of Avian Biology 49:jav-01447. DOI: 10.1111/jav.01447.

Rempel RS, Francis CM, Robinson JN, Campbell M. 2013. Comparison of audio recording system performance for detecting and monitoring songbirds. Journal of Field Ornithology 84:86-97. DOI: 10.1111/jofo.12008.

Sugai LSM, Silva TSF, Ribeiro JW, Llusia D. 2019. Terrestrial Passive Acoustic Monitoring: Review and Perspectives. BioScience 69:15-25. DOI: 10.1093/biosci/biy147.

Turgeon P, Van Wilgenburg S, Drake K. 2017. Microphone variability and degradation: implications for monitoring programs employing autonomous recording units. Avian Conservation and Ecology 12. DOI: 10.5751/ACE-00958-120109.

Whytock RC, Christie J. 2017. Solo: an open source, customizable and inexpensive audio recorder for bioacoustic research. Methods in Ecology and Evolution 8:308-312. DOI: 10.1111/2041-210X.12678.

Wickham H. 2009. Ggplot2: elegant graphics for data analysis. New York: Springer. 


\section{Table 1 (on next page)}

Microphone elements used in the study, along with letter codes used in Figure 1. MEMS: Microelectromechanical systems, Signal-to-noise ratios and sensitivity values provided by manufacturer. 


\begin{tabular}{|c|c|c|c|c|c|c|}
\hline Microphone element & Code & Type & Format & Manufacturer & $\left|\begin{array}{l}\text { Signal-to-noise } \\
\text { ratio } 1 \mathrm{kHz}(\mathrm{dB})\end{array}\right|$ & $\begin{array}{l}\text { Sensitivity } \\
\text { (dB SPL) }\end{array}$ \\
\hline POM-1345P-C3310-R & A & \multirow{7}{*}{$\begin{array}{l}\text { electret } \\
\text { condenser }\end{array}$} & \multirow[b]{5}{*}{ capsule } & \multirow[b]{5}{*}{ PUI Audio } & 55 & -45 \\
\hline POM-2735P-R & B & & & & 60 & -35 \\
\hline ROM-2235P-HD-R & C & & & & 68 & -35 \\
\hline POM-2730L-HD-R & $\mathrm{D}$ & & & & 74 & -30 \\
\hline AOM-5024L-HD-R & E & & & & 80 & -24 \\
\hline ICS-40720 & $\mathrm{F}$ & & MEMS & Invensense & 70 & -38 \\
\hline WM-61A & G & & capsule & Panasonic & 62 & -35 \\
\hline PMM-3738-VM1000-R & $\mathrm{H}$ & piezoelectric & \multirow[b]{3}{*}{ MEMS } & Vesper & 62 & -38 \\
\hline SPM0404UD5 & I & \multirow{4}{*}{$\begin{array}{l}\text { electret } \\
\text { condenser }\end{array}$} & & \multirow[b]{3}{*}{ Knowles } & 59 & -42 \\
\hline SPU0410LR5H-QB & $\mathrm{J}$ & & & & 63 & -38 \\
\hline FG-23629-C36 & $\mathrm{K}$ & & \multirow{2}{*}{ capsule } & & 66 & -53 \\
\hline EM258 & L & & & Primo & 74 & -32 \\
\hline
\end{tabular}




\section{Table 2 (on next page)}

Results for selecting the best model predicting measured signal-to-noise ratio with specified signal-to-noise ratio based on corrected Akaike Information Criterion 


\begin{tabular}{|l|r|r|l|}
\hline Model & Degrees of & & \\
& freedom & AICc & Formula (SNR in dB @ 1 kHz) \\
\hline Null & 2 & 182.43 & $\mathrm{SNR}_{\text {measured }} \sim 1$ \\
\hline Simple & 3 & 176.06 & $\mathrm{SNR}_{\text {measured }} \sim \mathrm{SNR}_{\text {specified }}$ \\
\hline Manufacturer & 8 & 159.12 & $\mathrm{SNR}_{\text {measured }} \sim \mathrm{SNR}_{\text {specified }}+$ Manufacturer \\
\hline Full & 9 & 165.04 & $\mathrm{SNR}_{\text {measured }} \sim \mathrm{SNR}_{\text {specified }}$ * Manufacturer \\
\hline
\end{tabular}

1 


\section{Table 3(on next page)}

Results for selecting the best model predicting detection spaces with measured signalto-noise ratio based on corrected Akaike Information Criterion 


\begin{tabular}{|c|c|c|c|c|}
\hline Frequency & Model & $\begin{array}{l}\text { Degrees of } \\
\text { freedom }\end{array}$ & AICc & Formula (area in $\mathrm{m}^{2}, \mathrm{SNR}$ in $\mathrm{dB}$ ) \\
\hline \multirow[b]{3}{*}{$1 \mathrm{kHz}$} & Linear & 3 & 367.18 & Detection area $\sim \mathrm{SNR}$ \\
\hline & Log-linear & 3 & 363.77 & Detection area $\sim \log (\mathrm{SNR})$ \\
\hline & Polynomial & 4 & 364.89 & Detection area $\sim$ poly $(\mathrm{SNR}, 2)$ \\
\hline \multirow[b]{3}{*}{$40 \mathrm{kHz}$} & Linear & 3 & 354.07 & Detection area $\sim \mathrm{SNR}$ \\
\hline & Log-linear & 3 & 355.81 & Detection area $\sim \log (\mathrm{SNR})$ \\
\hline & Polynomial & 4 & 356.21 & Detection area $\sim \operatorname{poly}(\mathrm{SNR}, 2)$ \\
\hline
\end{tabular}

1 


\section{Table 4 (on next page)}

Results for selecting the best model predicting bird and bat vocalisation activities with measured signal-to-noise ratio based on corrected Akaike Information Criterion.

We did not test a linear model for bats because the data were distinctly count-distributed. We included the interaction with the recording nights for bat models due to large differences between recording nights. 


\begin{tabular}{|c|c|c|c|c|}
\hline Taxon & Model & $\begin{array}{l}\text { Degrees of } \\
\text { freedom }\end{array}$ & AICc & $\begin{array}{l}\text { Formula } \\
\text { activity: min for birds, s for bats }\end{array}$ \\
\hline \multirow[b]{4}{*}{ Birds } & linear & 4 & 95.64 & total_activity $\sim \log (\mathrm{SNR}$ _dB $)+$ recording_date \\
\hline & GLM & 3 & 122.27 & $\begin{array}{l}\text { round(total_activity) } \sim \log \left(\mathrm{SNR} \_\mathrm{dB}\right)+ \\
\text { recording_date }\end{array}$ \\
\hline & $\begin{array}{l}\text { GLM negative } \\
\text { binomial }\end{array}$ & 4 & 125.35 & $\begin{array}{l}\text { round(total_activity) } \sim \log (\mathrm{SNR} \text { _dB })+ \\
\text { recording_date }\end{array}$ \\
\hline & GLM Gamma & 4 & 156.74 & $\begin{array}{l}\text { total_activity }+0.01 \sim \log \left(\mathrm{SNR} \_\mathrm{dB}\right)+ \\
\text { recording_date }\end{array}$ \\
\hline \multirow[b]{3}{*}{ Bats } & GLM & 4 & 338.79 & total_activity $\sim \mathrm{SNR}$ _dB $*$ recording.date \\
\hline & $\begin{array}{l}\text { GLM negative } \\
\text { binomial }\end{array}$ & 5 & 227.86 & total_activity $\sim$ SNR_dB $*$ recording.date \\
\hline & GLM Gamma & 5 & 235.11 & total_activity $\sim \mathrm{SNR}$ _dB $*$ recording.date \\
\hline
\end{tabular}

1 


\section{Figure 1}

Relationship between specified and measured microphone signal-to-noise ratio at $1 \mathrm{kHz}$ for different manufacturers and formats.

Letters identify microphone models (see Table 1). The dotted line indicates a 1:1 relationship, and solid lines indicate linear fits for each manufacturer with more than one microphone manufacturer. Microphone self-noise measurement protocols differ between measured and specified values. 


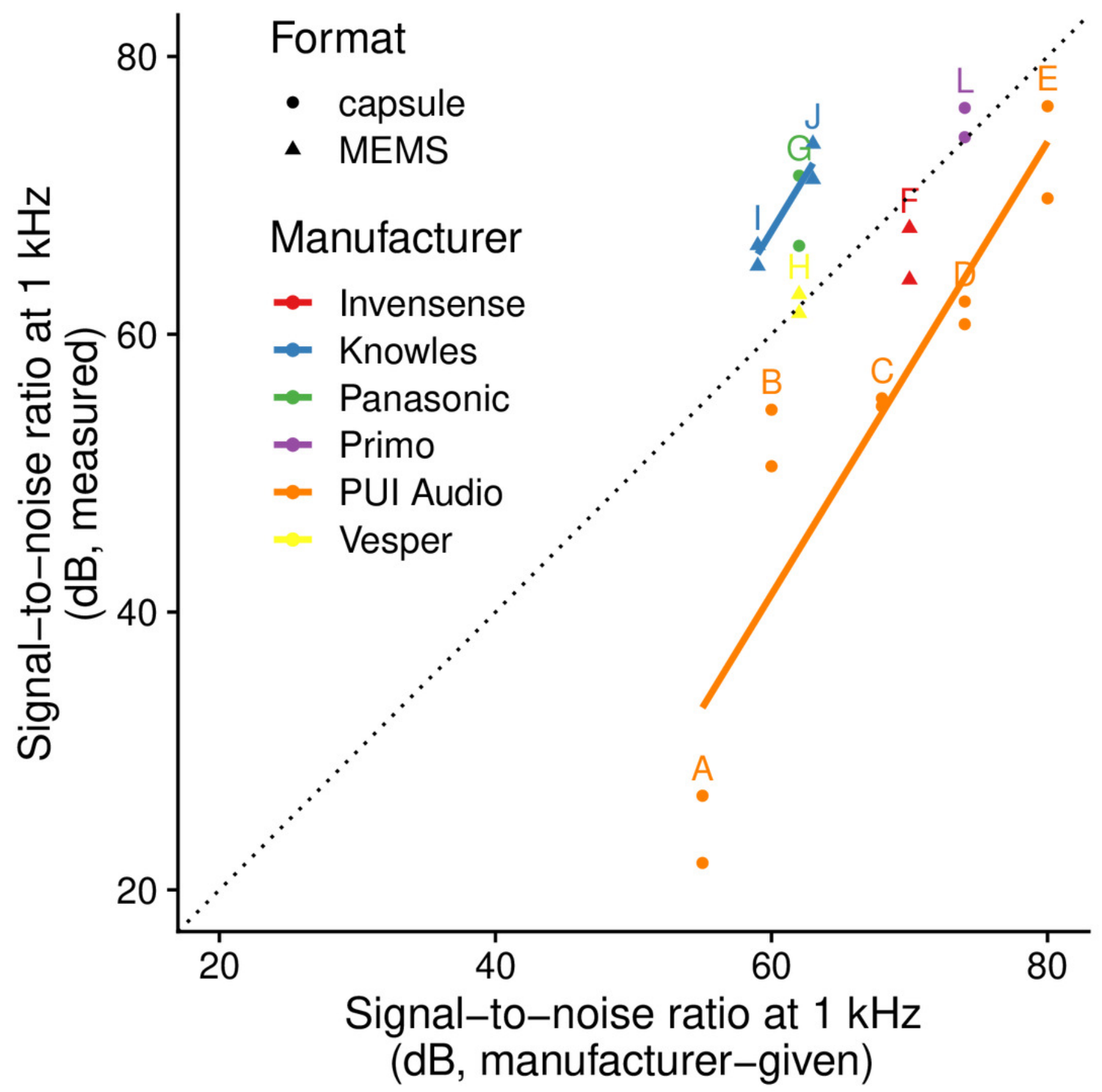


Figure 2

The influence of measured microphone signal-to-noise ratio on detection space areas in the audible $(1 \mathrm{kHz})$ and ultrasound $(40 \mathrm{kHz})$ ranges.

Lines show predictions from a linear regression against log-transformed (for birds) and nontransformed signal-to-noise ratio (for bats).

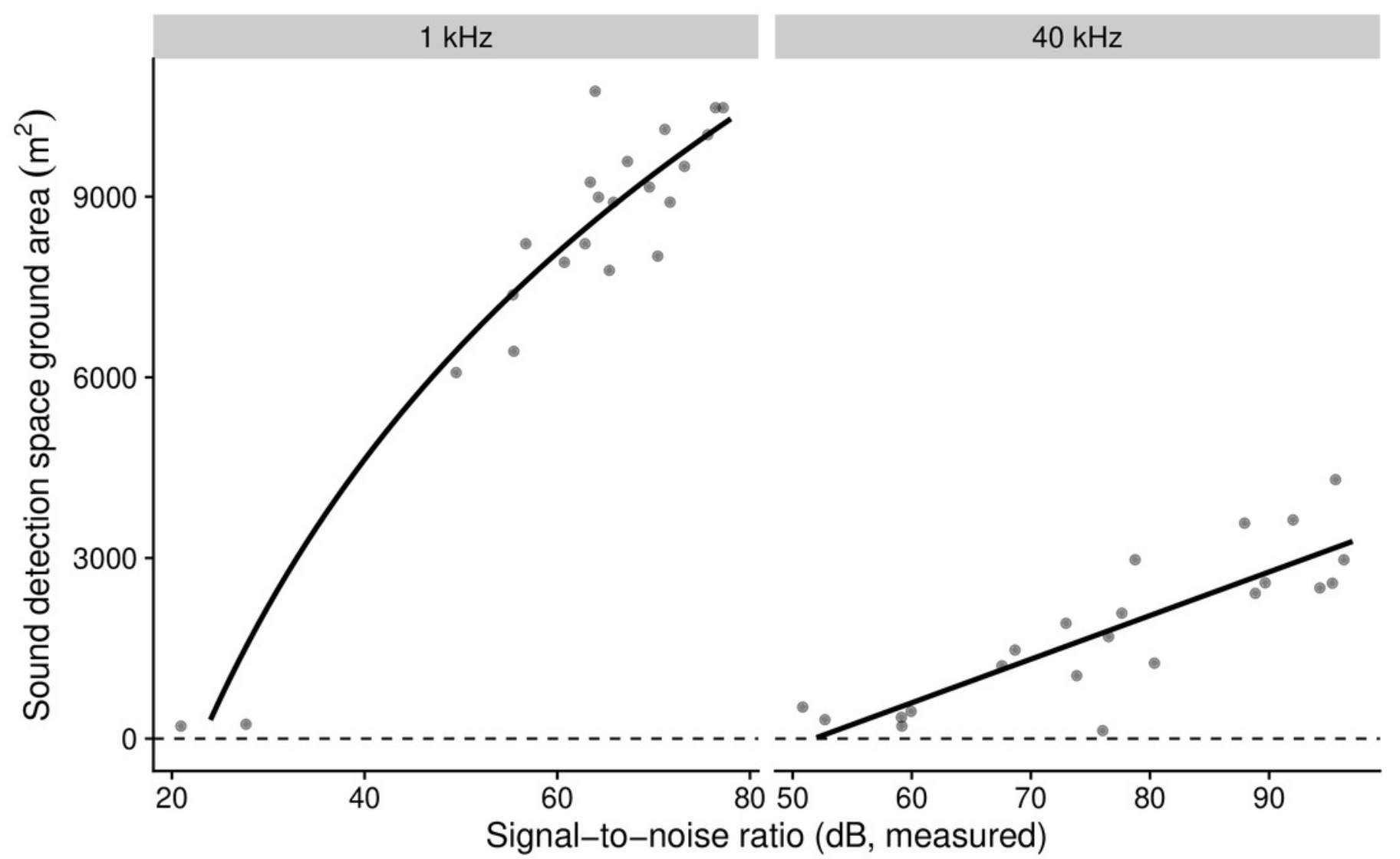


Figure 3

Vocalisation activity of birds and bats against microphone signal-to-noise ratio in the audible ( $1 \mathrm{kHz}$ for birds) and ultrasound (40 kHz for bats) ranges.

Lines show predictions from a generalised linear model with a negative binomial family (for bats) and a linear model using log-transformed signal-to-noise ratio (for birds). Sampling day was used as fixed effect.

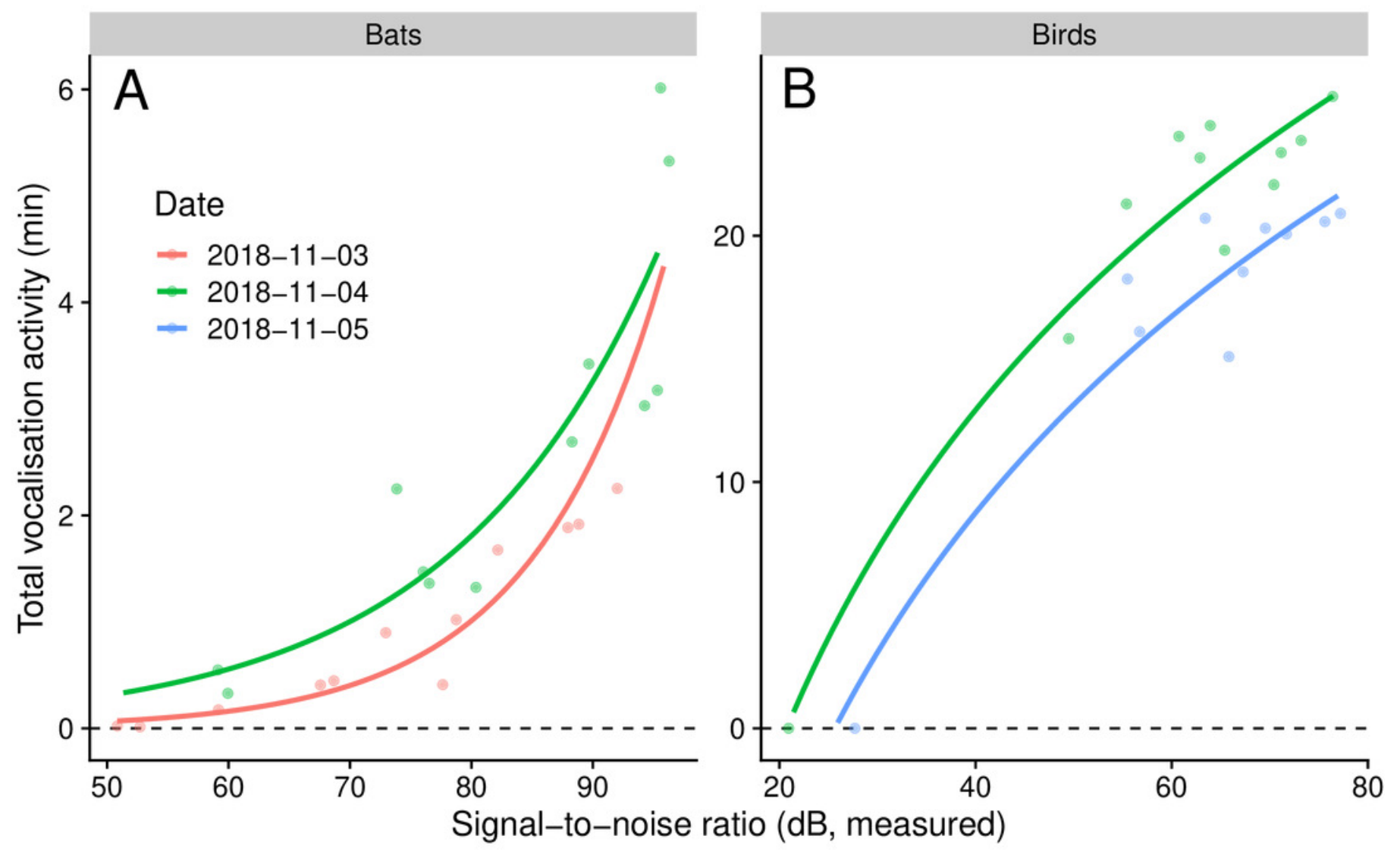


Figure 4

Species accumulation curves of each microphone, recording bat $(A)$ and bird $(B)$ species on different days, plotted against sampling time.

Signal-to-noise ratios are scaled within each taxon for achieving higher color contrast.
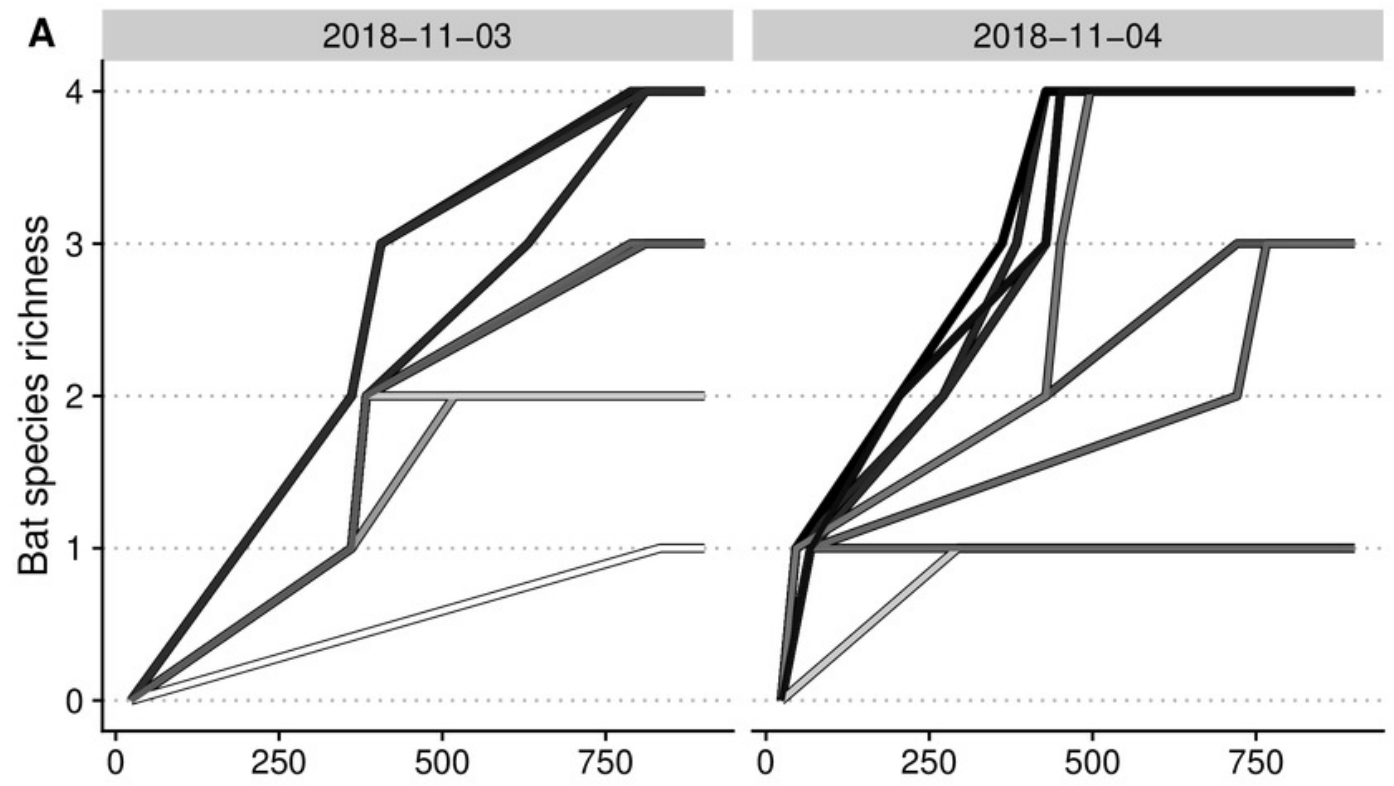

Signal-to-noise ratio @40 kHz (dB)

\section{0 80 70 60}

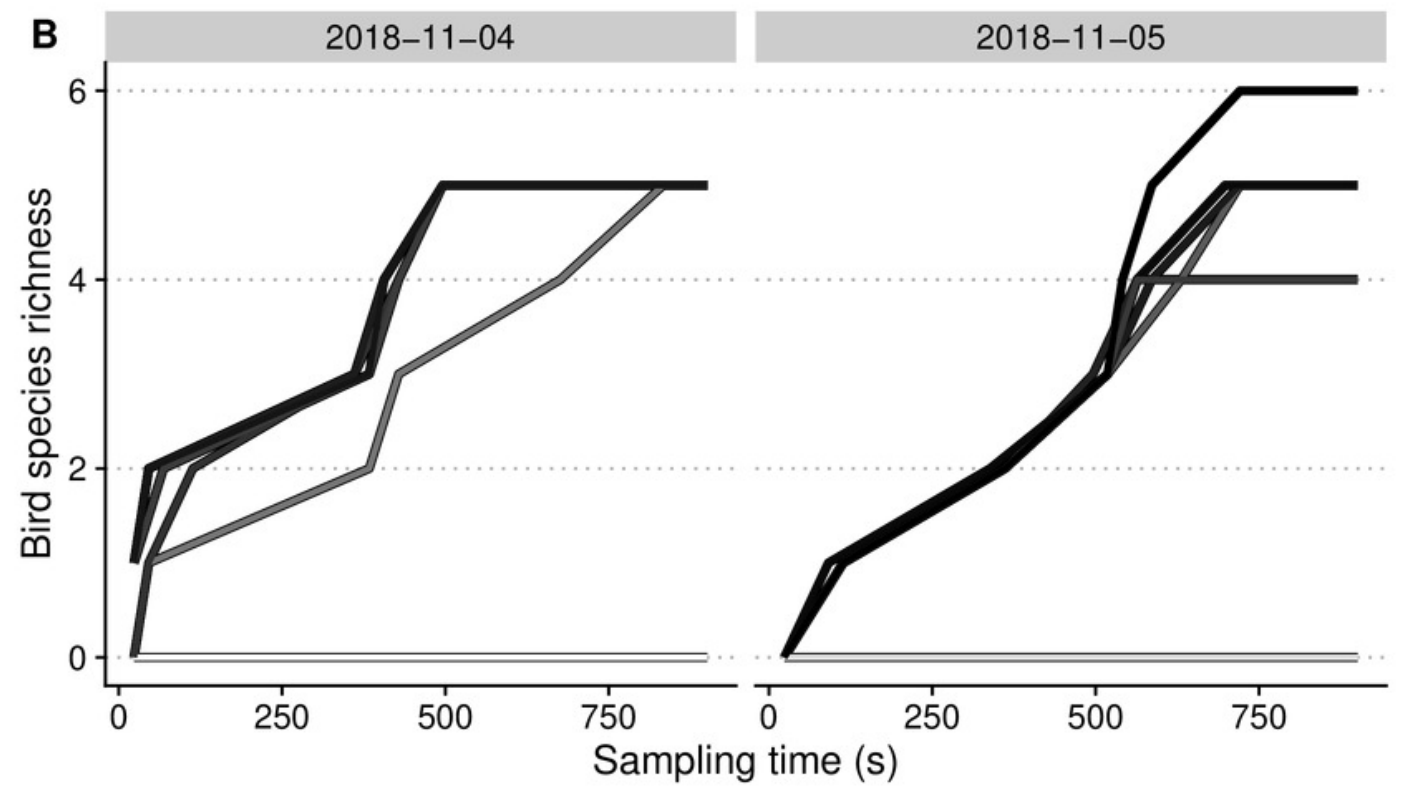

Signal-to-noise ratio @1 kHz (dB)

\section{0}

60

50

40

30 
Figure 5

Automated call detection performance for birds and bats for different days and microphone sets, measured by the total number of calls, true, and false positives, depending on recognizer score cutoff.

We defined acceptable precision and recall values with a value of 0.5 and show the range of recognizer score cutoffs where these minimal values are attained with green bars.

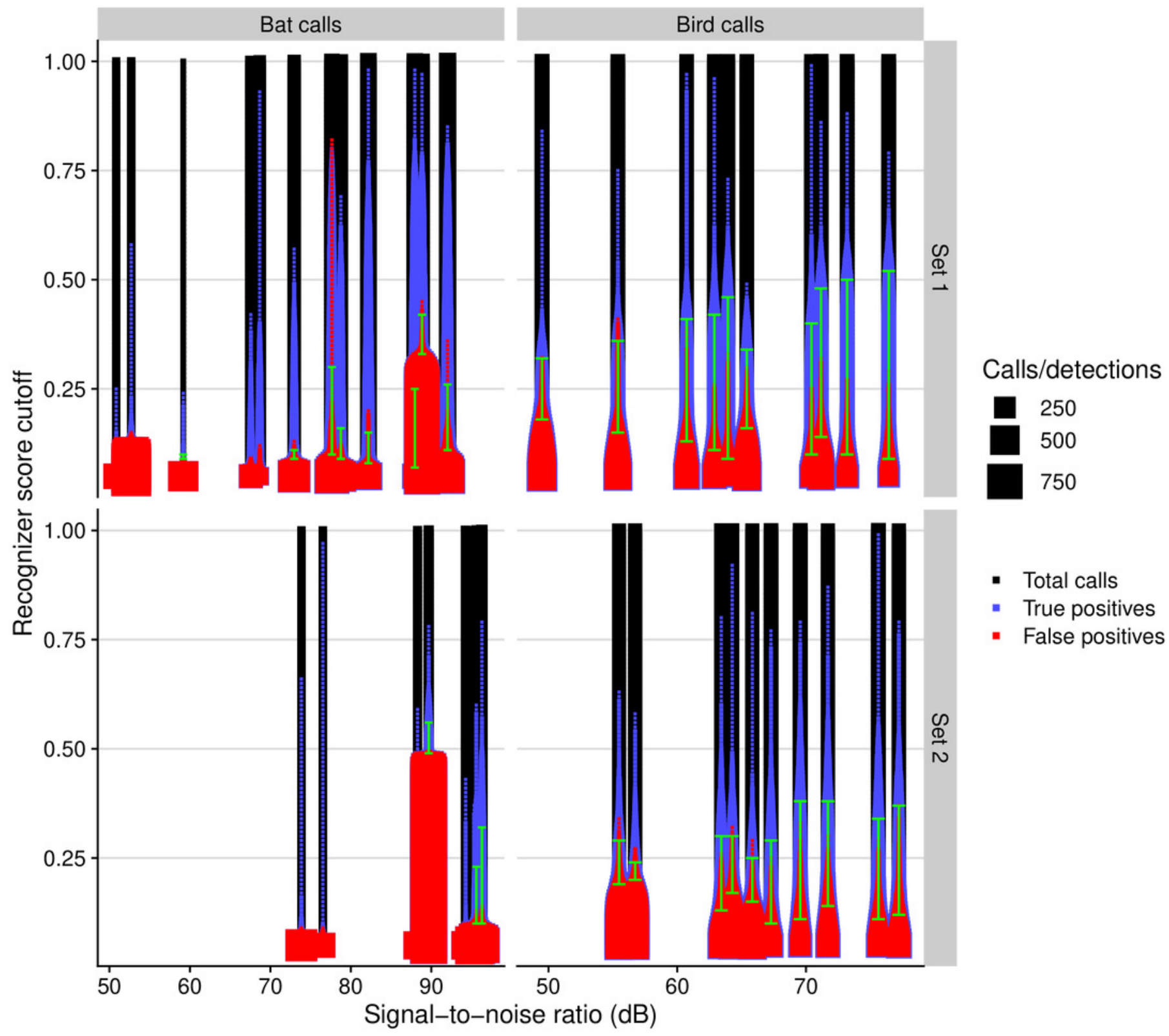

